\title{
DISCRETE-ORDINATES AND STREAMLINE DIFFUSION METHODS FOR A FLOW DESCRIBED BY BGK MODEL*
}

\author{
M. ASADZADEH ${ }^{\dagger}$, E. KAZEMI ${ }^{\ddagger}$,AND R. MOKHTARI ${ }^{\S}$
}

\begin{abstract}
A rarefied gas flow through a channel with arbitrary cross section is studied based on the linearized Bhatnagar-Gross-Krook model. The discrete velocity and streamline diffusion finite element methods are combined to yield a numerical scheme. For this scheme we derive stability and optimal convergence rates in the $L_{2}$-type norms. The optimality is due to the maximal available regularity of the exact solution for the corresponding hyperbolic PDE. The potential of the proposed, combined methods is illustrated with some numerical examples.
\end{abstract}

Key words. rarefied gas, linearized BGK model, discrete velocity, streamline diffusion, stability, convergence

AMS subject classifications. 65M15, 65M60, 76P05

DOI. $10.1137 / 120885747$

1. Introduction. In this paper we study the approximate solution for the flow of gas through a channel with arbitrary cross section, described by the linearized two-dimensional Bhatnagar-Gross-Krook (BGK) kinetic equation [4] using a discrete velocity model, in the discrete-ordinates setting approximating in velocity, combined with the streamline diffusion (SD) finite element discretization in the spatial variable.

The physical problem has diverse applications in, e.g., microelectromechanical systems and nanotechnology. Microducts, microturbines, or vacuum equipment are examples of small industrial devices involving the gas flow at an arbitrary Knudsen number. Since numerical solution of the Boltzmann equation in general geometry requires a six-dimensional phase space grid (three dimensions in each physical and velocity domains), the computational effort in full dimensions is seemingly involved. Nevertheless, in certain physical systems due to flow conditions, certain linearizations of the governing kinetic equation and reducing the number of space and velocity coordinates can be applicable. Therefore, in general, constructing effective numerical methods for this type of multidimensional problem is sought for physically relevant assumptions that might circumvent computational challenges. In this regard, and in the realm of the discrete-ordinates, the discrete velocity model has been developed as one of the most common techniques for the numerical solution of the space homogeneous Boltzmann equation and related kinetic models $[6,7]$. This method has been successfully applied to solve mixture problems [9] and also some more general

*Submitted to the journal's Computational Methods in Science and Engineering section July 23, 2012; accepted for publication (in revised form) May 19, 2014; published electronically August 14, 2014.

http://www.siam.org/journals/sisc/36-4/88574.html

$\dagger$ Department of Mathematics, Chalmers University of Technology and Göteborg University, SE412 96, Göteborg, Sweden (mohammad@chalmers.se.). This author's work was partially supported by the Swedish Foundation of Strategic Research (SSF) in the Gothenburg Mathematical Modeling Center (GMMC) and by the Swedish Research Council (VR).

${ }_{\ddagger}^{\ddagger}$ Department of Mathematics, Chalmers University of Technology and Göteborg University, SE412 96, Göteborg, Sweden, and Department of Mathematical Sciences, Isfahan University of Technology, Isfahan 84156-83111, Iran (ehsan_kfbl@yahoo.se).

$\S$ Department of Mathematical Sciences, Isfahan University of Technology, Isfahan 84156-83111, Iran (mokhtari@cc.iut.ac.ir).

B729 
models including polyatomic gases with chemical reactions [5]. The discrete velocity scheme is based on replacing the collision integral term by a certain quadrature sum and requires that the resulting discretized equations are valid only at discrete velocities corresponding to the numerical integration nodes. Then, the scheme is further discretized in the spatial variable by, e.g., a consistent finite-difference method. Additional difficulties arise for the domains with curved boundaries in space. In the conventional approach, the discretization in the spatial domain is usually based on regular mesh. This is the cause of the main difficulty when the method is applied to flow domains with irregular boundaries. To deal with such complex geometries it is more adequate to employ some version of the finite element approach which is based on unstructured mesh and can be readily applied to flows in complex spatial configurations. In this regard, e.g., the SD finite element method is a generalized version of the standard Galerkin method for the hyperbolic type problems having both good stability and high accuracy properties. The SD method, used for our purpose in this paper, is obtained by modifying the test function through adding a multiple (roughly, of the order of the mesh parameter) of the convective term involved in the equation. This gives a weighted least square control of the residual of the finite element solution. For a detailed description of the SD method for fluid problems, we refer to the monograph by Hughes and Brooks [13], and papers [3], [14], [15], and the references therein. We also point out that for transitional and nearly continuum flows, the stability condition of conventional finite-difference methods applied to the kinetic equation in the polar coordinate system results in a small time step which is proportional to the spatial mesh size. As a result the computational cost for determining near-continuum steady-state solutions may be unrealistically high and the results can only be presented for flows with rarefaction parameters up to at most 50 units. However, in the present work, based on finite element approximations, the mass flow rate for the Poiseuille flow in the channel is computed across the whole range of Knudsen numbers from the free-molecular to continuum regimes. To sum up, our objective is twofold:

(i) To construct and analyze numerical schemes for gas flow through a general class of spatial domains, including a wide diversity of channel forms, used in applications. This would require, e.g., a further development of the existing approaches for gas flow in symmetric geometries.

(ii) To develop a numerical scheme that improves stability without sacrificing accuracy and introduce a general approach, adopted to pressure-driven isothermal flows of rarefied gas in long micro channels (Poiseuille flow), and suitable for flows in complex spatial configurations in all regimes (with larger range for $\delta$ ) of the gas flow including the hydrodynamic, transitional, and free molecular ones.

An outline of this paper is as follows. Section 2 contains notation and preliminaries, where we also introduce the continuous kinetic model that we consider and describe some parameters used throughout the paper. Section 3 is devoted to the construction of some discrete velocity models as reasonable weak approximations for the Boltzmann collision operator. We also propose a scheme for spatial discretization based on the SD approach. Finally, we present some results concerning steady kinetic problems. In our concluding section 4 , we justify the theory by implementing some numerical examples.

2. Notation and preliminaries. Under certain physical constrains, the distribution of gas molecules can be described by the Boltzmann equation. Due to the number of unknown variables and hyperbolic structure of the equation the numerical solution of this equation is rather involved. One way out is to simplify the collision 
operators applying a kinetic model, as the one governed by the BGK equation, that is numerically accessible and yet preserves the main properties of the original equation. Such a model for a steady flow reads as follows (see, e.g., Cercignani [8]): find $\tilde{f}$ such that

$$
\boldsymbol{v} \cdot \nabla \tilde{f}(\boldsymbol{v}, \boldsymbol{x})=J(\tilde{f}),
$$

where $\boldsymbol{x}=(x, y, z)$ is the spatial variable and $\boldsymbol{v}=\left(v_{x}, v_{y}, v_{z}\right)$ is the velocity vector. Further

$$
J(\tilde{f})=\nu\left(\tilde{f}^{M}-\tilde{f}\right),
$$

and the gradient is taken with respect to the spatial variable $x, \nu$ is the collision frequency, and $\tilde{f}^{M}$ is the local Maxwellian distribution with the same density, velocity, and temperature as a gas having the distribution function $\tilde{f}$. The nonlinearity of the collision term is exhibited by the fact that the density, velocity, and temperature parameters all are functions of $\tilde{f}$. The BGK model possesses all the collisional invariants. Let the functions $\varphi_{i}, i=0, \ldots, 4$, be the collision invariants defined as $\varphi_{0}=1$, $\left(\varphi_{1}, \varphi_{2}, \varphi_{3}\right)=\boldsymbol{v}$, and $\varphi_{4}=|\boldsymbol{v}|^{2}=\left|\varphi_{1}\right|^{2}+\left|\varphi_{2}\right|^{2}+\left|\varphi_{3}\right|^{2}$. To derive a linearized version of the BGK equation, we consider $\tilde{f}$ to be defined as

$$
\tilde{f}=f_{0}(1+\varepsilon g),
$$

where $f_{0}$ is the absolute Maxwellian distribution which, by an appropriate choice of Galilean frame and mass and velocity units, can be assumed to be of the form

$$
f_{0}=\frac{1}{\pi^{3 / 2}} e^{-|v|^{2}}
$$

and $g$ is a certain function described below. Substituting (2.3) into (2.1), we obtain

$$
\boldsymbol{v} \cdot \nabla g=\nu_{0}\left[\sum_{i=0}^{4} \varphi_{i}^{\prime}\left(\varphi_{i}^{\prime}, g\right)_{f_{0}}-g\right],
$$

where we use the collision relation (2.2) and a weighted scalar product defined by

$$
(f, g)_{f_{0}}=\int_{\mathbb{R}^{3}} f(\boldsymbol{v}) g(\boldsymbol{v}) f_{0} d \boldsymbol{v} .
$$

Further, in (2.5), $\nu_{0}$ is the collision frequency at equilibrium, which is a constant; $\varphi_{i}^{\prime}, i=0, \ldots, 4$, are the collision invariants with $\varphi_{0}^{\prime}=1,\left(\varphi_{1}^{\prime}, \varphi_{2}^{\prime}, \varphi_{3}^{\prime}\right)=\sqrt{2} \boldsymbol{v}$, and $\varphi_{4}^{\prime}=\frac{1}{\sqrt{6}}\left(\left|\varphi_{1}^{\prime}\right|^{2}+\left|\varphi_{2}^{\prime}\right|^{2}+\left|\varphi_{3}^{\prime}\right|^{2}-3\right)=\sqrt{\frac{2}{3}}\left(|\boldsymbol{v}|^{2}-\frac{3}{2}\right)$, which are normalized using the scalar product (2.6). It can be easily seen that the collision operator, defined by

$$
\mathcal{C}(g)=\left[\sum_{i=0}^{4} \varphi_{i}^{\prime}\left(\varphi_{i}^{\prime}, g\right)_{f_{0}}-g\right],
$$

is symmetric with respect to the inner product (2.6) and satisfies the nonpositivity condition $(\mathcal{C}(g), g)_{f_{0}} \leq 0$. Further, we have

$$
\operatorname{Ker} \mathcal{C}=\left\{\sum_{i=0}^{4} c_{i} \varphi_{i}^{\prime}: c_{i} \in \mathbb{R}\right\} .
$$


The Poiseuille flow, i.e., the flow of a gas through a long channel having a restricted width induced by a density, temperature, or pressure gradient, and likewise the Couette flow, i.e., the flow of a gas between parallel plates induced by moving them with opposite velocities, can be modeled by the linearized BGK equation. For the sake of simplicity, the idea is explained for Poiseuille flows. We may single out the $z$-dependence and represent the function $g$ in (2.3) by

$$
g(x, y, z, \boldsymbol{v})=z+\psi(x, y, \boldsymbol{v}) .
$$

Now assuming that the flow is isothermal and the velocities normal to the walls are zero, we obtain the following equation for $\psi$ :

$$
v_{x} \frac{\partial \psi}{\partial x}+v_{y} \frac{\partial \psi}{\partial y}=\delta\left(2 v_{z} u-\psi(x, y, \boldsymbol{v})\right)-v_{z} .
$$

Here $\delta$ is the so-called rarefaction parameter, which in the present study coincides with the collision frequency $\nu_{0}$. Moreover,

$$
u(x, y)=\frac{1}{\pi^{\frac{3}{2}}} \int_{\mathbb{R}^{3}} v_{z} e^{-|\boldsymbol{v}|^{2}} \psi(x, y, \boldsymbol{v}) d \boldsymbol{v},
$$

and we have also assumed that

$$
\frac{1}{\pi^{\frac{3}{2}}} \int_{\mathbb{R}^{3}} e^{-|\boldsymbol{v}|^{2}} g d \boldsymbol{v}=z .
$$

These are typical assumptions in fluid dynamics for pressure-driven flows through long channels with small pressure gradient (cf. [18]). Multiplying (2.10) by $\frac{v_{z}}{\sqrt{\pi}} e^{-v_{z}^{2}}$ and integrating with respect to $v_{z}$ we obtain

$$
v_{x} \frac{\partial \phi}{\partial x}+v_{y} \frac{\partial \phi}{\partial y}=\delta\left(\tilde{u}-\phi\left(x, y, v_{x}, v_{y}\right)\right)-\frac{1}{2},
$$

where

$$
\phi\left(x, y, v_{x}, v_{y}\right)=\frac{1}{\sqrt{\pi}} \int_{\mathbb{R}} v_{z} e^{-v_{z}^{2}} \psi d v_{z},
$$

and $\tilde{u}$ (which remains identical to $u$ in (2.10)) corresponds to (2.11) with two-dimensional velocity and represents the bulk velocity defined as

$$
\tilde{u}(x, y) \equiv u(x, y)=\frac{1}{\pi} \int_{\mathbb{R}} \int_{\mathbb{R}} e^{-v_{x}^{2}-v_{y}^{2}} \phi\left(x, y, v_{x}, v_{y}\right) d v_{x} d v_{y} .
$$

Hence, we consider the two-dimensional integro-differential equation

$$
\boldsymbol{v} \cdot \nabla f(\boldsymbol{v}, \boldsymbol{x})=\delta \mathcal{C}(f)+S(\boldsymbol{v}, \boldsymbol{x}),
$$

associated with the inflow boundary condition

$$
f(\boldsymbol{v}, \boldsymbol{x})=0, \quad \boldsymbol{x} \in \Gamma_{\boldsymbol{v}}^{-} .
$$

The collision operator is given by

$$
\mathcal{C}(f)=\frac{1}{\pi} \int_{\mathbb{R}^{2}} f(\boldsymbol{v}, \boldsymbol{x}) e^{-|\boldsymbol{v}|^{2}} d \boldsymbol{v}-f(\boldsymbol{v}, \boldsymbol{x}),
$$

Copyright $@$ by SIAM. Unauthorized reproduction of this article is prohibited. 
and for each $\boldsymbol{v} \in \mathbb{R}^{2}$,

$$
\Gamma_{\boldsymbol{v}}^{-}=\{\boldsymbol{x} \in \partial \Omega: \mathbf{n} \cdot \boldsymbol{v}<0\} .
$$

Here, $\boldsymbol{x}=(x, y), \boldsymbol{v}=\left(v_{x}, v_{y}\right), \Omega$ is the spatial domain, and $\mathbf{n}$ is the outward unit normal to $\partial \Omega$ at the point $\boldsymbol{x} \in \partial \Omega$. As we mentioned earlier the two-component vectors $\boldsymbol{x}$ and $\boldsymbol{v}$, are defined in the cross section sheet of the channel. $S(\boldsymbol{v}, \boldsymbol{x})$ is an arbitrary source term, which, e.g., for recovery of the Poiseuille flow in the channel is a constant. Finally $\delta$, known as the rarefaction parameter, is an important dimensionless flow quantity which characterizes the rarefaction degree of the gas. The boundary condition represents particles departing from the wall. To proceed, we introduce the weighted scalar product

$$
(f, g)_{\hat{f}_{0}}=\int_{\mathbb{R}^{2}} f g \hat{f}_{0} d \boldsymbol{v}
$$

with the weight $\hat{f}_{0}(\boldsymbol{v})=\frac{1}{\pi} e^{-|\boldsymbol{v}|^{2}}$. Then, the collision operator $\mathcal{C}$ is symmetric with respect to this inner product. Moreover, $\operatorname{Ker} \mathcal{C}$ is nontrivial and $\mathcal{C}$ is strictly negative on the orthogonal complement of $\operatorname{Ker} \mathcal{C}$.

As a quantity of practical interest, used later on, we also introduce the dimensionless flow rate

$$
G=\frac{2}{|\Omega|} \int_{\Omega} u(\boldsymbol{x}) d \boldsymbol{x}
$$

with $|\Omega|$ being the area of the cross section and $u(\boldsymbol{x})$ the bulk velocity for (2.16), defined by

$$
u(\boldsymbol{x})=\frac{1}{\pi} \int_{\mathbb{R}^{2}} f(\boldsymbol{v}, \boldsymbol{x}) e^{-|\boldsymbol{v}|^{2}} d \boldsymbol{v}
$$

We set

$$
\langle f, g\rangle_{\Gamma_{v}^{ \pm}}=\int_{\Gamma_{v}^{ \pm}} f g(\boldsymbol{v} \cdot \mathbf{n}) d s
$$

where

$$
\Gamma_{\boldsymbol{v}}^{ \pm}=\{\boldsymbol{x} \in \Gamma:=\partial \Omega: \boldsymbol{v} \cdot \mathbf{n} \gtrless 0\} .
$$

Throughout the paper $C$ will denote a constant not necessarily the same at each occurrence and independent of all involved parameters and functions, unless otherwise specifically specified. By $(\cdot, \cdot)_{Q}$ we shall denote the usual $L_{2}(Q)$ scalar product and by $\|\cdot\|_{Q}$ the corresponding $L_{2}(Q)$-norm. Finally, we use the standard notation for Sobolev spaces together with their norms and seminorms (cf. [1]).

3. Discrete ordinates and the SD method. To construct the combined discrete-ordinates/SD scheme for the problem (2.16)-(2.19), we first focus on the velocity discretization and approximate the integral appearing in the collision term (2.18) using the certain quadrature rule. To this end, we write the numerical quadrature to be used in the form

$$
\int_{\mathbb{R}^{2}} F(\boldsymbol{v}) d \sigma \sim \sum_{\boldsymbol{v} \in \Delta} \omega_{\boldsymbol{v}} F(\boldsymbol{v})
$$


where $\omega_{v}$ are positive weights such that $\sum_{v \in \Delta} \omega_{v} \equiv 1$, and $\Delta$ is a discrete set of nodes defined below. Using the polar coordinate $\boldsymbol{v}=(c \cos \theta, c \sin \theta)$ with $c=|\boldsymbol{v}|$ in the quadrature rule above, we have that

$$
\int_{0}^{2 \pi} \int_{0}^{\infty} F(c, \theta) e^{-c^{2}} c d c d \theta \sim \sum_{v \in \Delta} \omega_{\boldsymbol{v}} F(\boldsymbol{v})
$$

Let now

$$
\Delta=\left\{\boldsymbol{v}_{i j}: \boldsymbol{v}_{i j}=c_{i}\left(\cos \left(\theta_{j}\right), \sin \left(\theta_{j}\right)\right), 1 \leq i \leq N, 1 \leq j \leq M\right\}
$$

be the quadrature set, with the uniform angular discretization $\theta_{j}=2 \pi j / M$, and the radial Gauss quadrature points $c_{i}=r_{i} / \sqrt{2}$, where $r_{i}$ are the zeros of Hermite orthogonal polynomials on $(0, \infty)$ associated with the distribution $d \sigma(r)=\frac{1}{2 \pi} r e^{-\frac{1}{2} r^{2}} d r$. The number of quadrature points in $\Delta$ is then $n=M N$. Our error analysis for the discrete-ordinates method works for the general quadrature rule for (2.22). For a quadrature rule of the form (3.1), which is exact for polynomials of degree $\leq m$, one can prove that (see [2])

$$
\left|\int_{\mathbb{R}^{2}} F(\boldsymbol{v}) d \sigma-\sum_{\boldsymbol{v} \in \Delta} \omega_{\boldsymbol{v}} F(\boldsymbol{v})\right| \leq C m^{-\alpha}\|F\|_{\alpha} \quad \forall F \in H^{\alpha}\left(\mathbb{R}^{2}\right), \quad \alpha \geq 1 .
$$

Note that $\alpha$, the maximal available regularity of $F$, can be noninteger. Then the optimal error estimates would require applying embedding techniques from the interpolation spaces.

The collision operator $\mathcal{C}$ in (2.18) can be approximated by the discretized operator $\hat{\mathcal{C}}$ given by

$$
\hat{\mathcal{C}}(f)=-f(c, \theta, \boldsymbol{x})+\sum_{i=1}^{N} \sum_{j=1}^{M} \omega_{i j} f\left(c_{i}, \theta_{j}, \boldsymbol{x}\right) .
$$

Using the quadrature rule (3.5), we may discretize (2.16) in the velocity space to get

$$
\begin{cases}\boldsymbol{v}_{i j} \cdot \nabla f^{i j}=\delta \hat{\mathcal{C}}\left(f^{i j}\right)+S^{i j} & \text { in } \Delta \times \Omega, \\ f^{i j}=0 & \text { on } \Gamma_{i j}^{-} .\end{cases}
$$

Here, $f^{i j}$ is an approximation of $f\left(\boldsymbol{v}_{i j}, \cdot\right)$ and $\Gamma_{i j}^{-}=: \Gamma_{\boldsymbol{v}_{i j}}^{-}$is the inflow boundary with $\boldsymbol{v}_{i j}$ defined as in (3.3). The equation system (3.6) is a first-order hyperbolic problem in the spatial domain, which will be further discretized by the SD method. We write $\boldsymbol{g}:=\left\{g^{i j}\right\}_{i, j}$ or simply $\boldsymbol{g}:=\left\{g^{i j}\right\}$ and define the subspace $W$ by

$$
W=\left\{\boldsymbol{g}: g^{i j}(\boldsymbol{x}) \in L^{2}(\Omega), \quad \boldsymbol{v}_{i j} \cdot \nabla g^{i j}(\boldsymbol{x}) \in L^{2}(\Omega), \text { with }\left.g^{i j}\right|_{\Gamma_{i j}^{-}}=0\right\} .
$$

For $\boldsymbol{f}, \boldsymbol{g} \in W$, we define the scalar product

$$
\langle\langle\boldsymbol{f}, \boldsymbol{g}\rangle\rangle=\sum_{i=1}^{N} \sum_{j=1}^{M} \omega_{i j} \int_{\Omega} f^{i j} g^{i j} d \boldsymbol{x} .
$$

Recalling the definition of the operator $\hat{\mathcal{C}}$, we find that for any two distribution functions $\boldsymbol{f}$ and $\boldsymbol{g} \in W$,

$$
\langle\langle\hat{\mathcal{C}}(\boldsymbol{f}), \boldsymbol{g}\rangle\rangle=\langle\langle\boldsymbol{f}, \hat{\mathcal{C}}(\boldsymbol{g})\rangle\rangle
$$

Copyright (c) by SIAM. Unauthorized reproduction of this article is prohibited. 
Now, by the Cauchy-Schwarz inequality and the fact that $\sum_{i, j} \omega_{i j} \leq 1$, we find that

$$
\begin{aligned}
\langle\langle\hat{\mathcal{C}}(\boldsymbol{f}), \boldsymbol{f}\rangle\rangle & =\sum_{i, j} \omega_{i j} \int_{\Omega} \hat{\mathcal{C}}\left(f^{i j}\right) f^{i j} d \boldsymbol{x} \\
& =\int_{\Omega}\left(-\sum_{i, j} \omega_{i j}\left(f^{i j}\right)^{2}+\sum_{i, j} \omega_{i j} f^{i j} \sum_{i, j} \omega_{i j} f^{i j}\right) d \boldsymbol{x} \leq 0 .
\end{aligned}
$$

The relation (3.10) may be referred as the nonpositivity property of the discrete collision operator. Hence, (3.9) and (3.10) indicate that the discrete collision operator $\hat{\mathcal{C}}$ is both symmetric and nonpositive. Let now $\hat{\Pi}$ be the orthogonal projection onto the complement of the kernel of the discrete collision operator $\hat{\mathcal{C}}$; then we have

$$
\hat{\mathcal{C}}(\boldsymbol{f})=\hat{\mathcal{C}}(\hat{\Pi} \boldsymbol{f}) .
$$

Using the definition of $\langle\langle\cdot, \cdot\rangle\rangle$ and (3.11), by applying the Cauchy-Schwarz inequality, we see that the operator $\hat{\mathcal{C}}$ is bounded in the space $W$; indeed, for all $\boldsymbol{f}, \boldsymbol{g} \in W$ we have

$$
-\langle\langle\hat{\mathcal{C}}(\boldsymbol{f}), \boldsymbol{g}\rangle\rangle \leq 2\langle\langle\hat{\Pi} \boldsymbol{f}, \hat{\Pi} \boldsymbol{f}\rangle\rangle^{1 / 2}\langle\langle\boldsymbol{g}, \boldsymbol{g}\rangle\rangle^{1 / 2} .
$$

In order to apply the finite element procedure we need to introduce a weak formulation for (3.6) by simply multiplying the equation by a test function $g$, in a certain suitable function space, and integrating over the spatial region. To construct the SD finite element method, we start with the standard Galerkin approach and modify the weak formulation, using test functions consisting of the sum of a test function $g$ (used in the standard Galerkin method) and an extra term of the form $\gamma(\boldsymbol{v} \cdot \nabla g)$, i.e., a convective term in the direction of the streamlines. It is the scalar product of this additional term, in the test function, with the conversion term in the equation itself that results to an artificial diffusion (hence, the name of the method streamline diffusion). In this way we employ different test and trial function spaces, which in the discrete version both will have the same finite dimension. Then, for (3.6), we define continuous variational formulation as follows: find $\left\{f^{i j}\right\} \in W$ such that for all $\left\{g^{i j}\right\} \in W$,

$$
\begin{aligned}
& \left(\boldsymbol{v}_{i j} \cdot \nabla f^{i j}, g^{i j}+\gamma_{i j}\left(\boldsymbol{v}_{i j} \cdot \nabla g^{i j}\right)\right)_{\Omega}-\delta\left(\hat{\mathcal{C}}\left(f^{i j}\right), g^{i j}+\gamma_{i j}\left(\boldsymbol{v}_{i j} \cdot \nabla g^{i j}\right)\right)_{\Omega} \\
& \quad=\left(S^{i j}, g^{i j}+\gamma_{i j}\left(\boldsymbol{v}_{i j} \cdot \nabla g^{i j}\right)\right)_{\Omega},
\end{aligned}
$$

where $\gamma_{i j}$ is a positive parameter which, for the hyperbolic problems usually, is of the order of the mesh size. Multiplying by quadrature weights and summing over the quadrature set, the semidiscrete method, for the velocity discretization, (3.13) can be written as

$$
\begin{aligned}
& \sum_{i, j} \omega_{i j}\left\{\left(\boldsymbol{v}_{i j} \cdot \nabla f^{i j}, g^{i j}+\gamma_{i j}\left(\boldsymbol{v}_{i j} \cdot \nabla g^{i j}\right)\right)_{\Omega}-\delta\left(\hat{\mathcal{C}}\left(f^{i j}\right), g^{i j}+\gamma_{i j}\left(\boldsymbol{v}_{i j} \cdot \nabla g^{i j}\right)\right)_{\Omega}\right\} \\
& =\sum_{i, j} \omega_{i j}\left(S^{i j}, g^{i j}+\gamma_{i j}\left(\boldsymbol{v}_{i j} \cdot \nabla g^{i j}\right)\right)_{\Omega} .
\end{aligned}
$$

As for the spatial discretization we let $\mathcal{C}_{h}=\{K\}$ be a finite element subdivision of $\Omega$ into the elements $K$ with the mesh parameter $h=\operatorname{diam} K$. Further, let $P_{k}(K)$ be the set of polynomials of degree at most $k$ on $K$ in $\boldsymbol{x}$ and define the finite element space of continuous piecewise polynomials, viz.,

$$
V_{h}=\left\{g: g \text { is continuous, } g \in P_{k}(K), \forall K \in \mathcal{C}_{h}\right\} .
$$


We also define the finite element space associated to the semidiscrete scheme by

$$
\boldsymbol{V}_{h}=\left\{\left\{g^{i j}\right\} \in\left(V_{h}\right)^{N M}:\left.g^{i j}\right|_{\Gamma_{i j}^{-}}=0\right\}
$$

and introduce the corresponding bilinear form defined for all $\boldsymbol{f}_{h}, \boldsymbol{g} \in \boldsymbol{V}_{h}$ as

$$
\begin{aligned}
B\left(\boldsymbol{f}_{h}, \boldsymbol{g}\right)= & \sum_{i, j} \omega_{i j}\left\{\left(\boldsymbol{v}_{i j} \cdot \nabla f_{h}^{i j}, g^{i j}+\gamma_{i j}\left(\boldsymbol{v}_{i j} \cdot \nabla g^{i j}\right)\right)_{\Omega}\right. \\
& \left.-\delta\left(\hat{\mathcal{C}}\left(f_{h}^{i j}\right), g^{i j}+\gamma_{i j}\left(\boldsymbol{v}_{i j} \cdot \nabla g^{i j}\right)\right)_{\Omega}\right\}
\end{aligned}
$$

Now the objective is to solve the following fully discrete variational problem: find $f_{h} \in V_{h}$ such that

$$
B\left(\boldsymbol{f}_{h}, \boldsymbol{g}\right)=L(\boldsymbol{g}) \quad \forall \boldsymbol{g} \in \boldsymbol{V}_{h},
$$

where $L$ is the linear form defined by the right-hand side of (3.14):

$$
L(\boldsymbol{g})=\sum_{i, j} \omega_{i j}\left(S^{i j}, g^{i j}+\gamma_{i j}\left(\boldsymbol{v}_{i j} \cdot \nabla g^{i j}\right)\right)_{\Omega} .
$$

For the method (3.18), we derive stability (coercivity) estimates and error bounds in the following triple norm $\||\cdot|\|$, defined over $\boldsymbol{V}_{h}$ :

$$
\|\boldsymbol{g}\| \|^{2}=\sum_{i, j} \omega_{i j}\left(\left\|\sqrt{\gamma_{i j}}\left(\boldsymbol{v}_{i j} \cdot \nabla g^{i j}\right)\right\|_{\Omega}^{2}+\delta\left\|\hat{\Pi} g^{i j}\right\|_{\Omega}^{2}+\int_{\Gamma_{i j}+}\left(g^{i j}\right)^{2}\left(\boldsymbol{v}_{i j} \cdot \mathbf{n}\right) d s\right) .
$$

Here $\hat{\Pi}$ is the orthogonal projection onto the complement of the kernel of collision operator in $\boldsymbol{V}_{h}$ as defined in (3.11). From (3.10), since the space $\boldsymbol{V}_{h}$ is finite dimensional, we may state that there exists a positive constant $\hat{\lambda}_{0}$ such that

$$
\hat{\lambda}_{0}\langle\langle\hat{\Pi} \boldsymbol{f}, \hat{\Pi} \boldsymbol{f}\rangle\rangle \leq-\langle\langle\hat{\mathcal{C}}(\boldsymbol{f}), \boldsymbol{f}\rangle\rangle .
$$

Below we shall show that the bilinear form $B$ is coercive.

Lemma 3.1. Assume that the SD parameter $\gamma_{i j}$ satisfies

$$
\gamma_{i j} \sim \frac{\hat{\lambda}_{0}}{\delta}
$$

then there exists a constant $\alpha>0$, depending on $\hat{\lambda}_{0}$, such that

$$
B(\boldsymbol{g}, \boldsymbol{g}) \geq \alpha\|\boldsymbol{g} \mid\|^{2} \quad \forall \boldsymbol{g} \in \boldsymbol{V}_{h} .
$$

Proof. We let $\boldsymbol{f}^{h}=\boldsymbol{g}$ in (3.17); then

$$
\begin{aligned}
B(\boldsymbol{g}, \boldsymbol{g})= & \sum_{i, j} \omega_{i j}\left\{\left(\boldsymbol{v}_{i j} \cdot \nabla g^{i j}, g^{i j}+\gamma_{i j}\left(\boldsymbol{v}_{i j} \cdot \nabla g^{i j}\right)\right)_{\Omega}\right. \\
& \left.-\delta\left(\hat{\mathcal{C}}\left(g^{i j}\right), g^{i j}+\gamma_{i j}\left(\boldsymbol{v}_{i j} \cdot \nabla g^{i j}\right)\right)_{\Omega}\right\} .
\end{aligned}
$$

Using Green's formula and the zero inflow boundary condition we get

$$
\left(\boldsymbol{v}_{i j} \cdot \nabla g^{i j}, g^{i j}\right)_{\Omega}=\frac{1}{2} \int_{\Gamma_{i j}^{+}}\left(g^{i j}\right)^{2}\left(\boldsymbol{v}_{i j} \cdot \mathbf{n}\right) d \boldsymbol{v} .
$$

Copyright $@$ by SIAM. Unauthorized reproduction of this article is prohibited. 
Further, by (3.21) we have

$$
-\sum_{i, j} \omega_{i j}\left(\hat{\mathcal{C}}\left(g^{i j}\right), g^{i j}\right)_{\Omega} \geq \hat{\lambda}_{0} \sum_{i, j} \omega_{i j}\left\|\hat{\Pi} g^{i j}\right\|_{\Omega}^{2},
$$

and using (3.12) yields

$$
\begin{aligned}
& \sum_{i, j} \omega_{i j}\left(\hat{\mathcal{C}}\left(g^{i j}\right), \gamma_{i j}\left(\boldsymbol{v}_{i j} \cdot \nabla g^{i j}\right)\right)_{\Omega} \\
& \quad \leq 2\left(\sum_{i, j} \omega_{i j}\left\|\hat{\Pi} g^{i j}\right\|_{\Omega}^{2}\right)^{\frac{1}{2}}\left(\sum_{i, j} \omega_{i j}\left\|\sqrt{\gamma_{i j}}\left(\boldsymbol{v}_{i j} \cdot \nabla g^{i j}\right)\right\|_{\Omega}^{2}\right)^{\frac{1}{2}} .
\end{aligned}
$$

Hence, we deduce that

$$
\sum_{i, j} \omega_{i j} \delta\left(\hat{\mathcal{C}}\left(g^{i j}\right), \gamma_{i j}\left(\boldsymbol{v}_{i j} \cdot \nabla g^{i j}\right)\right)_{\Omega} \leq \sum_{i, j} \omega_{i j}\left(\hat{\lambda}_{0} \delta\left\|\hat{\Pi} g^{i j}\right\|_{\Omega}^{2}+\frac{\delta}{\hat{\lambda}_{0}}\left\|\sqrt{\gamma_{i j}}\left(\boldsymbol{v}_{i j} \cdot \nabla g^{i j}\right)\right\|_{\Omega}^{2}\right) .
$$

Choosing $\gamma_{i j}$ as in (3.22), the relations (3.24)-(3.28) yield the desired result.

In what follows, we shall use the following interpolation error estimates (see Ciarlet [10]): let $f \in H^{r+1}(\Omega)$; then there exists an interpolant, $\tilde{f}_{h} \in V_{h}$, of $f$ such that

$$
\begin{aligned}
\left\|f-\tilde{f}_{h}\right\|_{\Omega} & \leq C h^{r+1}\|f\|_{r+1, \Omega}, \\
\left\|f-\tilde{f}_{h}\right\|_{1, \Omega} & \leq C h^{r}\|f\|_{r+1, \Omega}, \\
\left\|f-\tilde{f}_{h}\right\|_{\partial \Omega} & \leq C h^{r+1 / 2}\|f\|_{r+1, \Omega} .
\end{aligned}
$$

Let $\left\{\eta^{i j}\right\}=\left\{f^{i j}\right\}-\left\{\tilde{f}_{h}^{i j}\right\}$ be the interpolation error and set $\left\{\xi^{i j}\right\}=\boldsymbol{f}_{h}-\left\{\tilde{f}_{h}^{i j}\right\}$. We may write the error as

$$
\left\{e^{i j}\right\}=\left\{f^{i j}\right\}-\boldsymbol{f}_{h}=\left\{\eta^{i j}\right\}-\left\{\xi^{i j}\right\} .
$$

The convergence theorem, for the spatial discretization, is now as follows.

THEOREM 3.2. Let $\left\{f^{i j}\right\}$ and $\boldsymbol{f}_{h}$ be the solutions of the continuous and discrete problem satisfying (3.13) and (3.18), respectively. If the SD parameter $\gamma_{i j}$ satisfies

$$
\gamma_{i j} \sim \min \left\{\frac{\hat{\lambda}_{0}}{\delta}, \frac{h}{\left\|\boldsymbol{v}_{i j}\right\|_{\infty}}\right\}
$$

then there is a constant $C=C(\Omega)$ such that

$$
\left|\left\|\left\{f^{i j}\right\}-\boldsymbol{f}_{h}\right\|\right| \leq C h^{k+1 / 2}\left(\sum_{i, j} \omega_{i j}\left(\delta+\left\|\boldsymbol{v}_{i j}\right\|_{\infty}\right)\left\|f^{i j}\right\|_{k+1, \Omega}^{2}\right)^{1 / 2} .
$$

Proof. Using the triangle inequality we have

$$
\left\|\left|\{ e ^ { i j } \} \| | \leq | \| \{ \eta ^ { i j } \} \left\|\left|+\left\|\mid\left\{\xi^{i j}\right\}\right\| \| .\right.\right.\right.\right.
$$

The definition of the triple norm ||$|\cdot|||$ and the interpolation error estimates (3.29)(3.31) yield

$$
\left\|\left|\left\{\eta^{i j}\right\} \|\right| \leq C h^{k+1 / 2}\left(\sum_{i, j} \omega_{i j}\left(\delta+\left\|\boldsymbol{v}_{i j}\right\|_{\infty}\right)\left\|f^{i j}\right\|_{k+1, \Omega}^{2}\right)^{1 / 2} .\right.
$$

Copyright (c) by SIAM. Unauthorized reproduction of this article is prohibited. 
Using the Galerkin orthogonality relation $B\left(\left\{e^{i j}\right\},\left\{\xi^{i j}\right\}\right)=0$, since $\gamma_{i j}$ in (3.33) fulfills (3.22), we have that

$$
\begin{aligned}
\left.\alpha\|\mid\| \xi^{i j}\right\} \|\left.\right|^{2} \leq B\left(\left\{\xi^{i j}\right\},\left\{\xi^{i j}\right\}\right)= & B\left(\left\{\eta^{i j}\right\}-\left\{e^{i j}\right\},\left\{\xi^{i j}\right\}\right)=B\left(\left\{\eta^{i j}\right\},\left\{\xi^{i j}\right\}\right) \\
= & \sum_{i, j} \omega_{i j}\left\{\left(\boldsymbol{v}_{i j} \cdot \nabla \eta^{i j}, \xi^{i j}+\gamma_{i j}\left(\boldsymbol{v}_{i j} \cdot \nabla \xi^{i j}\right)\right)_{\Omega}\right. \\
& \left.-\delta\left(\hat{\mathcal{C}}\left(\eta^{i j}\right), \xi^{i j}+\gamma_{i j}\left(\boldsymbol{v}_{i j} \cdot \nabla \xi^{i j}\right)\right)_{\Omega}\right\} .
\end{aligned}
$$

Integrating by parts and using the fact that $\operatorname{div} v \equiv 0$, by the Cauchy-Schwarz inequality we obtain

$$
\begin{aligned}
\left(\boldsymbol{v}_{i j} \cdot \nabla \eta^{i j}, \xi^{i j}\right)_{\Omega}= & -\left(\eta^{i j}, \boldsymbol{v}_{i j} \cdot \nabla \xi^{i j}\right)_{\Omega}+\frac{1}{2} \int_{\Gamma_{i j}^{+}} \eta^{i j} \xi^{i j}\left(\boldsymbol{v}_{i j} \cdot \mathbf{n}\right) d s \\
\leq & \left\|\frac{1}{\sqrt{\gamma_{i j}}} \eta^{i j}\right\|_{\Omega}^{2}+\frac{1}{4}\left\|\sqrt{\gamma_{i j}} \boldsymbol{v}_{i j} \cdot \nabla \xi^{i j}\right\|_{\Omega}^{2} \\
& +\int_{\Gamma_{i j}^{+}}\left|\eta^{i j}\right|^{2}\left(\boldsymbol{v}_{i j} \cdot \mathbf{n}\right) d s+\frac{1}{8} \int_{\Gamma_{i j}^{+}}\left|\xi^{i j}\right|^{2}\left(\boldsymbol{v}_{i j} \cdot \mathbf{n}\right) d s
\end{aligned}
$$

Similarly, to bound the second term in (3.37), we have using (3.12), the CauchySchwarz inequality, and (3.9) that

$$
-\sum_{i, j} \omega_{i j} \delta\left(\hat{\mathcal{C}}\left(\eta^{i j}\right), \xi^{i j}\right)_{\Omega} \leq \sum_{i, j} \omega_{i j}\left\{C \delta\left\|\eta^{i j}\right\|_{\Omega}^{2}+\frac{\delta}{4}\left\|\hat{\Pi} \xi^{i j}\right\|_{\Omega}^{2}\right\}
$$

and

$$
-\sum_{i, j} \omega_{i j} \delta\left(\hat{\mathcal{C}}\left(\eta^{i j}\right), \gamma_{i j}\left(\boldsymbol{v}_{i j} \cdot \nabla \xi^{i j}\right)\right)_{\Omega} \leq \sum_{i, j} \omega_{i j}\left\{C \delta\left\|\hat{\Pi} \eta^{i j}\right\|_{\Omega}^{2}+\frac{\delta}{4 \hat{\lambda}_{0}}\left\|\gamma_{i j}\left(\boldsymbol{v}_{i j} \cdot \nabla \xi^{i j}\right)\right\|_{\Omega}^{2}\right\} .
$$

Combining the estimates (3.37)-(3.40) and using (3.33) we end up with

$$
\begin{aligned}
\left\|\mid\left\{\xi^{i j}\right\}\right\| \|^{2} \leq & B(\eta, \xi) \leq \frac{1}{4}\left\|\left\{\xi^{i j}\right\}\right\| \|^{2} \\
& +C \sum_{i, j} \omega_{i j}\left[\left\|\frac{1}{\sqrt{\gamma_{i j}}} \eta^{i j}\right\|_{\Omega}^{2}+\left\|\sqrt{\gamma_{i j}}\left(\boldsymbol{v}_{i j} \cdot \nabla \eta^{i j}\right)\right\|_{\Omega}^{2}+\int_{\Gamma_{i j}^{+}}\left|\eta^{i j}\right|^{2}\left(\boldsymbol{v}_{i j} \cdot \mathbf{n}\right) d s\right] .
\end{aligned}
$$

Using (3.29)-(3.31), a kick-back argument, and (3.33), we deduce that

$$
\left\|\left\{\xi^{i j}\right\}\right\| \mid \leq C h^{k+1 / 2}\left(\sum_{i, j} \omega_{i j}\left(\delta+\left\|\boldsymbol{v}_{i j}\right\|_{\infty}\right)\left\|f^{i j}\right\|_{k+1, \Omega}^{2}\right)^{1 / 2} .
$$

Inserting the inequalities (3.36) and (3.42) into (3.35) we obtain the desired result.

To proceed for fully discrete error estimates we invoke the contribution from the quadrature error. In this approach we decompose the global error as

$$
\left\{f_{h}^{i j}\right\}-\left\{f\left(\boldsymbol{v}_{i j}, \cdot\right)\right\}=\left\{f_{h}^{i j}\right\}-\left\{f^{i j}\right\}+\left\{f^{i j}\right\}-\left\{f\left(\boldsymbol{v}_{i j}, \cdot\right)\right\} .
$$

Copyright (c) by SIAM. Unauthorized reproduction of this article is prohibited. 
Then, by the triangle inequality,

$$
\left\|||\left\{f_{h}^{i j}\right\}-\left\{f\left(\boldsymbol{v}_{i j}, \cdot\right)\right\}\left|\left\|\leq\left|\left\|\left\{f_{h}^{i j}\right\}-\left\{f^{i j}\right\}\right\|\right|||||\left\{f^{i j}\right\}-\left\{f\left(\boldsymbol{v}_{i j}, \cdot\right)\right\}\right\|\right| .\right.
$$

We now need to bound the term $\left\|\left|\left\{f^{i j}\right\}-\left\{f\left(\boldsymbol{v}_{i j}, \cdot\right)\right\}\right|\right\|$ which is related to the quadrature formula (3.1). To this end, we consider (2.16) with $\boldsymbol{v}=\boldsymbol{v}_{i j}$ and subtract the result from (3.6) to obtain

$$
\boldsymbol{v}_{i j} \cdot \nabla \chi^{i j}-\delta \hat{\mathcal{C}}\left(\chi^{i j}\right)=\rho(\boldsymbol{x}), \quad \chi^{i j}=0 \text { on } \Gamma_{i j}^{-},
$$

where $\chi^{i j}=f^{i j}-f\left(\boldsymbol{v}_{i j}, \cdot\right)$ and

$$
\rho(\boldsymbol{x})=\frac{1}{\pi} \int f(\boldsymbol{v}, \boldsymbol{x}) e^{-|\boldsymbol{v}|^{2}} d \boldsymbol{v}-\sum_{i, j} \omega_{i j} f\left(\boldsymbol{v}_{i j}, \boldsymbol{x}\right)
$$

Using the scalar product introduced in (2.6), and multiplying (3.45) by $\left\{\gamma_{i j}\left(\boldsymbol{v}_{i j}\right.\right.$. $\left.\left.\nabla \chi^{i j}\right)\right\}$, we get that

$$
\begin{aligned}
& \left\langle\left\langle\left\{\boldsymbol{v}_{i j} \cdot \nabla \chi^{i j}\right\},\left\{\gamma_{i j}\left(\boldsymbol{v}_{i j} \cdot \nabla \chi^{i j}\right)\right\}\right\rangle\right\rangle-\delta\left\langle\left\langle\left\{\hat{\mathcal{C}}\left(\chi^{i j}\right)\right\},\left\{\gamma_{i j}\left(\boldsymbol{v}_{i j} \cdot \nabla \chi^{i j}\right)\right\}\right\rangle\right\rangle \\
& \quad=\left\langle\left\langle\rho,\left\{\gamma_{i j}\left(\boldsymbol{v}_{i j} \cdot \nabla \chi^{i j}\right)\right\}\right\rangle\right\rangle .
\end{aligned}
$$

Now the Cauchy-Schwarz inequality, combined with (3.12) and (3.33), yields

$$
\sum_{i, j} \omega_{i j} \gamma_{i j}\left\|\boldsymbol{v}_{i j} \cdot \nabla \chi^{i j}\right\|_{\Omega}^{2} \leq C\left(\|\rho(x)\|_{\Omega}^{2}+\delta^{2} \sum_{i, j} \omega_{i j}\left\|\hat{\Pi} \chi^{i j}\right\|_{\Omega}^{2}\right) .
$$

Similarly, taking the inner product of (3.45) with $\left\{\chi^{i j}\right\}$, applying the Green's theorem, and using the boundary condition in (3.45) we obtain

$$
\frac{1}{2} \sum_{i, j} \omega_{i j} \int_{\Gamma_{i j}^{+}}\left(\chi^{i j}\right)^{2} \boldsymbol{v}_{i j} \cdot \mathbf{n} d s-\sum_{i, j} \omega_{i j} \delta \int_{\Omega} \hat{\mathcal{C}}\left(\chi^{i j}\right) \chi^{i j} d \boldsymbol{x}=\sum_{i, j} \omega_{i j} \int_{\Omega} \chi^{i j} \rho d \boldsymbol{x}
$$

By a further use of the Cauchy-Schwarz inequality and (3.21) we deduce that

$$
\frac{1}{2} \sum_{i, j} \omega_{i j} \int_{\Gamma_{i j}^{+}}\left(\chi^{i j}\right)^{2} \boldsymbol{v}_{i j} \cdot \mathbf{n} d s+\delta \sum_{i, j} \omega_{i j}\left\|\hat{\Pi} \chi^{i j}\right\|_{\Omega}^{2} \leq C Z\|\rho\|_{\Omega},
$$

where $Z=\max _{i, j}\left\|\chi^{i j}\right\|_{\Omega}$. It remains to bound the term $\|\rho\|_{\Omega}$. To this end the quadrature error (3.4), applied to $\rho$, gives

$$
|\rho(\boldsymbol{x})| \leq C m^{-\alpha}\|f(\cdot, \boldsymbol{x})\|_{\alpha},
$$

and then square integration over $\Omega$ yields

$$
\|\rho\|_{\Omega}^{2} \leq C m^{-2 \alpha} \int_{\Omega}\|f(\cdot, \boldsymbol{x})\|_{\alpha}^{2} d \boldsymbol{x} .
$$

Finally, combining the estimates (3.48), (3.50), and (3.52) we get

$$
\begin{aligned}
\left\|\mid\left\{f^{i j}\right\}-\left\{f\left(\boldsymbol{v}_{i j}, \boldsymbol{x}\right)\right\}\right\| \|^{2} \leq & C m^{-2 \alpha} \int_{\Omega}\|f(\cdot, \boldsymbol{x})\|_{\alpha}^{2} d \boldsymbol{x} \\
& +C \delta Z m^{-\alpha}\left(\int_{\Omega}\|f(\cdot, \boldsymbol{x})\|_{\alpha}^{2} d \boldsymbol{x}\right)^{1 / 2} .
\end{aligned}
$$

Copyright $@$ by SIAM. Unauthorized reproduction of this article is prohibited. 
Based on these estimates and the spatial discretization error derived in Theorem 3.1 , now we formulate the fully discrete error estimate as the main result of this paper.

THEOREM 3.3. Let $f$ be the exact solution satisfying (2.16)-(2.19). Assume that $f \in H^{\alpha}\left(\mathbb{R}^{2}, H^{k+1}(\Omega)\right)$ and the quadrature approximation (3.4) is valid. Then, there is a constant $C>0$, independent of $h$ and $m$, such that for the fully discrete problem (3.18)-(3.19) the following estimate holds true:

$$
\begin{gathered}
\left\|\left\{f_{h}^{i j}(\boldsymbol{x})\right\}-\left\{f\left(\boldsymbol{v}_{i j}, \boldsymbol{x}\right)\right\}\right\|\left\|^{2} \leq C h^{2 k+1} \sum_{i, j} \omega_{i j}\left(\delta+\left\|\boldsymbol{v}_{i j}\right\|_{\infty}\right)\right\| f^{i j} \|_{k+1, \Omega}^{2} \\
+m^{-2 \alpha} \int_{\Omega}\|f(\cdot, \boldsymbol{x})\|_{\alpha}^{2} d \boldsymbol{x}+\left(\delta Z m^{-\alpha}\right)\left(\int_{\Omega}\|f(\cdot, \boldsymbol{x})\|_{\alpha}^{2} d \boldsymbol{x}\right)^{1 / 2} .
\end{gathered}
$$

Proof. Using the triangle inequality (3.44), Theorem 3.2 and the estimate (3.53) yield the desired result.

Remark 3.4. Here are some features of the problem (2.16)-(2.19): lack of an absorption term in (2.16) yields to stability with no explicit $L_{2}$-norm control. Actually we obtain the $L_{2}$-norm stability of orthogonal projection onto the complement of the kernel of the discrete collision operator. By some transformation (an exponential variable substitution), we may obtain an equation having an absorption term, and then the kernel of the integral operator will be changed. We may also consider some properties of the operator $\boldsymbol{v}_{i j} \cdot \nabla$ leading to an $L_{2}(\Omega)$ estimate. Since $\nabla \cdot \boldsymbol{v}_{i j}=0$, the convection operator $\boldsymbol{v}_{i j} \cdot \nabla: W \rightarrow L_{2}(\Omega)$ is an isomorphism (see, e.g., [11] and the references therein) and we have that

$$
\alpha\|g\|_{\Omega} \leq\left\|\boldsymbol{v}_{i j} \cdot \nabla g\right\|_{\Omega} \quad \forall g \in W
$$

for some constant $\alpha>0$. Note that in all the above estimates the seminorm $\left(L_{2^{-}}\right.$ norm of partial derivatives) appears with a coefficient of order $\mathcal{O}(\sqrt{h})$. Combining this and Theorem 3.3, the coefficients $\gamma_{i j}$ will appear in the $L_{2}$-norm estimates as well. Therefore, in implementations, one should expect to get an actual rate of convergence of order $\mathcal{O}\left(h^{k}+m^{-\alpha} h^{-\frac{1}{2}}\right)$, i.e., a rate reduced by the order of $h^{1 / 2}$, compared to the theory. Based on this phenomena, we may remove the last term in (3.54) and improve the error estimate. As a result of Theorem 3.3 and Remark 3.4, we may obtain an order of convergence for the mass flow rate $G$ introduced by (2.21).

Corollary 3.5. Assume that $G$ is the mass flow rate defined by (2.21), and its approximation $G_{h}$ is given by

$$
G_{h}=\frac{1}{|\Omega|} \sum_{i, j} \omega_{i j} \int_{\Omega} f_{h}^{i j}(\boldsymbol{x}) d \boldsymbol{x} .
$$

Then we have

$$
\left|G-G_{h}\right| \sim C_{\delta}^{-1} \mathcal{O}\left(\delta^{1 / 2} h^{k+1 / 2}+m^{-\alpha}+\delta^{1 / 2} m^{-\alpha / 2}\right),
$$

where $C_{\delta}=\min \left\{\delta, \frac{1}{\delta}, \gamma_{i j}\right\}$.

Proof. By the triangle inequality we may write

$$
\left|G-G_{h}\right| \leq|G-\hat{G}|+\left|\hat{G}-G_{h}\right|,
$$

Copyright (c) by SIAM. Unauthorized reproduction of this article is prohibited. 
where

$$
\hat{G}=\frac{1}{|\Omega|} \sum_{i, j} \omega_{i j} \int_{\Omega} f\left(\boldsymbol{v}_{i j}, \boldsymbol{x}\right) d \boldsymbol{x} .
$$

Applying the quadrature error (3.4), for the first term in (3.58) we get

$$
|G-\hat{G}| \leq C m^{-\alpha} \int_{\Omega}\|f(\cdot, \boldsymbol{x})\|_{\alpha} d \boldsymbol{x} .
$$

In addition, using Hölder inequality, for the second term in (3.58) we obtain

$$
\left|\hat{G}-G_{h}\right| \leq \sum_{i, j} \omega_{i j}\left\|f\left(\boldsymbol{v}_{i j}, \cdot\right)-f_{h}^{i j}\right\|_{\Omega} .
$$

Now, by Theorem 3.3 and Remark 3.4, we deduce that

$$
\left|\hat{G}-G_{h}\right| \sim C_{\delta}^{-1} \mathcal{O}\left(\delta^{1 / 2} h^{k+1 / 2}+m^{-\alpha}+\delta^{1 / 2} m^{-\alpha / 2}\right),
$$

where $C_{\delta}=\min \left\{\delta, \frac{1}{\delta}, \gamma_{i j}\right\}$. Then inserting (3.60) and (3.62) into (3.58), and using the regularity assumption on $f$, we obtain the desired result.

4. Numerical investigations. To justify the theoretical results, in this section we compute the mass flow rates in the wide range of rarefaction parameter $\delta$ for rectangular, triangular, and circular cross sections. The results are compared with those in the literature [16], [17], [20], [21]. We also investigate the performance of the proposed scheme on numerical convergence order in the norm $\||\cdot|\|$ defined by (3.20). For problem (2.16), and considering circular, rectangular, and triangular cross sections, Tables 1-3 show the results of performance of the combined scheme in computing the flow rate $\mathrm{G}$ introduced in (2.21). Tables $4-6$ concern justification of the optimal spatial convergence rate for the continuous piecewise linear approximation $(k=1$, $M \mathcal{O}\left(h^{k+1 / 2}\right) \sim h^{3 / 2}$ ) for Example 4.1 considered in the square domain.

Below we describe the details of the computational procedure: we have used piecewise linear polynomials with the mesh size $h=0.07$, combined with the quadrature formula with $M=15$ and $N=15$, i.e., 225 ordinates, to dicretize the problem (2.16). Since $-\hat{\mathcal{C}}$ is a symmetric positive semidefinite operator, the parameter $\hat{\lambda}_{0}$ in (3.33) may be approximated by the Rayleigh quotient from (3.21),

$$
\hat{\lambda}_{0} \leq \min _{\hat{\Pi} f \neq 0} \frac{-\langle\langle\hat{\mathcal{C}}(\boldsymbol{f}), \boldsymbol{f}\rangle\rangle}{\langle\langle\hat{\Pi} f, \hat{\Pi} \boldsymbol{f}\rangle\rangle}
$$

and thus by choosing $f^{i j}=1$ in $\boldsymbol{f}=\left\{f^{i j}\right\}$ for each $i, j$, we get $\hat{\lambda}_{0} \leq \min _{i, j}\left(1-\omega_{i j}\right)$, where $\omega_{i j}$ are the weights in the quadrature formula (3.1). We choose $\gamma_{0}$ of the SD parameter, $\gamma=\gamma_{0} h$, to be equals to 0.01 for $\delta \leq 5$, and 0.001 for $\delta>5$, subject to the constraint (3.33). In general, rarefaction enhances the diffusivity nature and hence, moderately larger values for $\delta$ would need only smaller SD modification, i.e., smaller $\gamma$ and $\gamma_{0}$. For $\delta<10$ the resulting linear algebraic system of equations is solved using a successive overrelaxation solver and the stopping criterion on the convergence of the iterative procedure is set equal to $10^{-12}$. Since for $\delta \gg 1$ the convergence of the iteration method slows down, for this case we have employed the direct method in solving the discretized systems. In Table 1 we present the results of calculating the flow rate $G$ in rectangular channels and compare these results with the corresponding 
TABLE 1

Reduced flow rate $G$ in rectangular channels versus rarefaction parameter $\delta$ and aspect ratio a/b.

\begin{tabular}{|c|c|c|c|c|c|c|}
\hline \multirow{2}{*}{$\delta$} & \multicolumn{6}{|c|}{$\mathrm{G}$} \\
\cline { 2 - 7 } & \multicolumn{2}{|c|}{$a / b=1$} & \multicolumn{2}{c|}{$a / b=2$} & \multicolumn{2}{c|}{$a / b=10$} \\
\cline { 2 - 7 } & {$[17,18]$} & $(3.18)$ & {$[18]$} & $(3.18)$ & {$[18]$} & $(3.18)$ \\
\hline .01 & 0.8281 & 0.8201 & 1.137 & 1.1229 & 1.910 & 1.9407 \\
.02 & 0.8213 & 0.8160 & 1.125 & 1.1145 & 1.858 & 1.8896 \\
.05 & 0.8076 & 0.8055 & 1.099 & 1.0944 & 1.759 & 1.7880 \\
.1 & 0.7934 & 0.7925 & 1.073 & 1.0716 & 1.665 & 1.6876 \\
.2 & 0.7766 & 0.7765 & 1.046 & 1.0462 & 1.563 & 1.5785 \\
.5 & 0.7622 & 0.7603 & 1.026 & 1.0243 & 1.454 & 1.4568 \\
.8 & 0.7614 & 0.7609 & 1.031 & 1.0288 & 1.425 & 1.4246 \\
1 & 0.7678 & 0.7652 & 1.041 & 1.0377 & 1.424 & 1.4213 \\
1.5 & 0.7861 & 0.7827 & 1.074 & 1.0700 & 1.448 & 1.4419 \\
2 & 0.8076 & 0.8056 & 1.115 & 1.1100 & 1.491 & 1.4834 \\
5 & 0.9885 & 0.9787 & 1.413 & 1.3977 & 1.870 & 1.8633 \\
10 & 1.329 & 1.3161 & 1.955 & 1.9559 & 2.599 & 2.5981 \\
15 & 1.705 & 1.6591 & 2.511 & 2.5166 & 3.357 & 3.3636 \\
20 & 2.000 & 2.0066 & 3.077 & 3.0831 & 4.121 & 4.1405 \\
30 & & 2.7071 & & 4.2219 & & 5.7088 \\
40 & & 3.4102 & & 5.3616 & & 7.2861 \\
50 & & 4.1136 & & 6.4991 & & 8.8677 \\
100 & & 7.6136 & & 12.1268 & & 16.7988 \\
200 & & 14.4824 & & 23.0328 & & 32.6890 \\
\hline
\end{tabular}

TABLE 2

Reduced flow rate $G$ in elliptical channels versus rarefaction parameter $\delta$ and aspect ratio a/b.

\begin{tabular}{|c|c|c|c|c|c|c|c|c|}
\hline \multirow{2}{*}{$\delta$} & \multicolumn{9}{|c|}{$\mathrm{G}$} \\
\cline { 2 - 8 } & \multicolumn{2}{|c|}{$a / b=1$} & \multicolumn{2}{|c|}{$a / b=1.1$} & \multicolumn{2}{c|}{$a / b=2$} & \multicolumn{2}{c|}{$a / b=10$} \\
\cline { 2 - 9 } & {$[16]$} & $(3.18)$ & {$[12]$} & $(3.18)$ & {$[12]$} & $(3.18)$ & {$[12]$} & $(3.18)$ \\
\hline .01 & 1.4760 & 1.4650 & 1.548 & 1.5360 & 2.066 & 2.0025 & 3.314 & 3.3414 \\
.02 & 1.4598 & 1.4538 & 1.529 & 1.5237 & 2.015 & 1.9802 & 3.198 & 3.2208 \\
.05 & 1.4295 & 1.4280 & 1.497 & 1.4958 & 1.985 & 1.9335 & 2.994 & 3.0048 \\
.1 & 1.4026 & 1.4018 & 1.469 & 1.4680 & 1.935 & 1.8912 & 2.817 & 2.8169 \\
.2 & 1.3816 & 1.3790 & 1.446 & 1.4447 & 1.893 & 1.8587 & 2.651 & 2.6427 \\
.5 & 1.3864 & 1.3835 & 1.499 & 1.4525 & 1.881 & 1.8777 & 2.534 & 2.5158 \\
.8 & 1.4252 & 1.4214 & 1.499 & 1.4958 & 1.951 & 1.9473 & 2.565 & 2.5419 \\
1 & 1.4583 & 1.4540 & 1.536 & 1.5323 & 2.009 & 2.0040 & 2.614 & 2.5877 \\
1.5 & 1.5532 & 1.5477 & 1.641 & 1.6365 & 2.170 & 2.1629 & 2.780 & 2.7457 \\
2 & 1.6576 & 1.6507 & 1.757 & 1.7505 & 2.344 & 2.3345 & 2.976 & 2.9337 \\
5 & 2.3483 & 2.3301 & 2.516 & 2.5138 & 3.471 & 3.4677 & 4.331 & 4.3242 \\
10 & 3.5633 & 3.5619 & 3.850 & 3.8488 & 5.430 & 5.4299 & 6.738 & 6.7717 \\
20 & 6.0411 & 6.0453 & 6.565 & 6.5689 & 9.402 & 9.4135 & 11.65 & 9.2620 \\
30 & 8.5333 & 8.5416 & 9.294 & 9.3015 & 13.39 & 13.4086 & 16.58 & 16.7387 \\
40 & 11.0295 & 11.0378 & 12.03 & 12.0334 & 17.39 & 17.3983 & 21.52 & 21.6815 \\
50 & 13.5269 & 13.5303 & 14.76 & 14.7608 & 21.38 & 21.3777 & 26.46 & 26.5811 \\
100 & 26.0214 & 25.9028 & & 28.2975 & & 41.0770 & & 50.4005 \\
200 & 51.0254 & 50.1348 & & 54.8049 & & 79.4233 & & 94.8108 \\
\hline
\end{tabular}

data from [17] and [18] for different aspect ratios $a / b$, where $2 a$ is the maximum tube length in the $x$ direction and $2 b$ is the maximum tube length in the $y$ direction. In Table 2 the computed results for the circular and elliptical cross sections are presented. These results are generally in good (within 1\%) agreement with the published results in [12] and [16] over the entire $\delta$ range. Some exceptions appear for the cases $\delta \gg 1$ and $\delta \ll 1$, where the difference between the results is slightly larger than $1 \%$. This is due to the presence of $\delta$ in approximation error formula (3.54). We may use the 
TABLE 3

Reduced flow rate $G$ in various triangular channels versus rarefaction parameter $\delta$.

\begin{tabular}{|c|c|c|c|c|c|c|}
\hline \multirow{2}{*}{$\delta$} & \multicolumn{6}{|c|}{$\mathrm{G}$} \\
\cline { 2 - 7 } & \multicolumn{2}{|c|}{ Equilateral } & \multicolumn{2}{|c|}{ Scalene } & \multicolumn{2}{|c|}{ Orthogonal isosceles } \\
\cline { 2 - 7 } & {$[20]$} & $(3.18)$ & {$[20]$} & $(3.18)$ & {$[20]$} & $(3.18)$ \\
\hline 0.001 & 0.929 & 0.9109 & 0.935 & 0.9162 & 0.965 & 0.9464 \\
0.01 & 0.919 & 0.9054 & 0.925 & 0.9106 & 0.954 & 0.9400 \\
0.1 & 0.872 & 0.8692 & 0.878 & 0.8734 & 0.902 & 0.8985 \\
0.5 & 0.831 & 0.8277 & 0.835 & 0.8303 & 0.854 & 0.8501 \\
0.75 & 0.829 & 0.8259 & 0.833 & 0.8279 & 0.851 & 0.8465 \\
1 & 0.834 & 0.8301 & 0.837 & 0.8317 & 0.854 & 0.8494 \\
1.5 & 0.851 & 0.8475 & 0.855 & 0.8484 & 0.870 & 0.8651 \\
2 & 0.876 & 0.8711 & 0.879 & 0.8716 & 0.894 & 0.8877 \\
5 & 1.06 & 1.0581 & 1.07 & 1.0584 & 1.08 & 1.0744 \\
10 & 1.41 & 1.4083 & 1.42 & 1.4090 & 1.43 & 1.4278 \\
20 & 2.14 & 2.1408 & 2.14 & 2.1443 & 2.17 & 2.1709 \\
30 & 2.88 & 2.8858 & 2.89 & 2.8929 & 2.92 & 2.9278 \\
40 & 3.62 & 3.6350 & 3.63 & 3.6456 & 3.67 & 3.6889 \\
50 & 4.37 & 4.3857 & 4.38 & 4.3994 & 4.43 & 4.4512 \\
100 & & 8.1352 & & 8.1565 & & 8.2501 \\
200 & & 15.5503 & & 15.5442 & & 15.7193 \\
\hline
\end{tabular}

TABLE 4

Error $\left\|u-u_{h}\right\|$ for Example 4.1 .

\begin{tabular}{|c|c|c|c|c|c|}
\hline$h$ & $\gamma_{0}=0$ & $\gamma_{0}=0.001$ & $\gamma_{0}=0.01$ & $\gamma_{0}=0.1$ & $\gamma_{0}=0.5$ \\
\hline \hline$\frac{\sqrt{2}}{5}$ & 0.247206982 & 0.244798006 & 0.227213803 & 0.178063742 & 0.216327675 \\
$\frac{\sqrt{2}}{10}$ & 0.026715009 & 0.02652664 & 0.025652036 & 0.030466757 & 0.0759276 \\
$\frac{\sqrt{2}}{15}$ & 0.011670016 & 0.01150648 & 0.010821089 & 0.013734404 & 0.045281935 \\
$\frac{\sqrt{2}}{20}$ & 0.006162771 & 0.006065313 & 0.005766944 & 0.008593496 & 0.032996712 \\
\hline
\end{tabular}

TABLE 5

Error $\left\|u-u_{h}\right\| \|$ for Example 4.1 .

\begin{tabular}{|c|c|c|c|c|c|}
\hline$h$ & $\gamma_{0}=0$ & $\gamma_{0}=0.001$ & $\gamma_{0}=0.01$ & $\gamma_{0}=0.1$ & $\gamma_{0}=0.5$ \\
\hline \hline$\frac{\sqrt{2}}{5}$ & 0.303022935 & 0.304333116 & 0.316307008 & 0.420452474 & 0.718022365 \\
$\frac{\sqrt{2}}{10}$ & 0.029895327 & 0.032654345 & 0.051285278 & 0.132921852 & 0.284534964 \\
$\frac{\sqrt{2}}{15}$ & 0.013185436 & 0.014826665 & 0.025414045 & 0.070742315 & 0.16144478 \\
$\frac{\sqrt{2}}{20}$ & 0.006925584 & 0.008218338 & 0.01572285 & 0.045652177 & 0.107539983 \\
\hline
\end{tabular}

TABLE 6

Error $\left|G-G_{h}\right|$ for Example 4.1.

\begin{tabular}{|c|c|c|c|c|c|}
\hline$h$ & $\gamma_{0}=0$ & $\gamma_{0}=0.001$ & $\gamma_{0}=0.01$ & $\gamma_{0}=0.1$ & $\gamma_{0}=0.5$ \\
\hline \hline$\frac{\sqrt{2}}{5}$ & 0.086942268 & 0.086250263 & 0.080363851 & 0.044469892 & 0.007591975 \\
$\frac{\sqrt{2}}{10}$ & 0.006840345 & 0.006843534 & 0.007019878 & 0.007931986 & 0.007860636 \\
$\frac{\sqrt{2}}{15}$ & 0.003764336 & 0.003679156 & 0.003300136 & 0.003239473 & 0.004556734 \\
$\frac{\sqrt{2}}{20}$ & 0.001084447 & 0.00109074 & 0.001217363 & 0.001589597 & 0.002807666 \\
\hline
\end{tabular}

higher-order quadrature rule and a finer mesh parameter in the physical domain for the cases of $\delta \ll 1$ and $\delta \gg 1$, respectively. Table 3 is for flows in triangular channels. The computed results are compared with those in [20], which are only presented for the $\delta$-values up to $\delta=50$.

Copyright $@$ by SIAM. Unauthorized reproduction of this article is prohibited. 


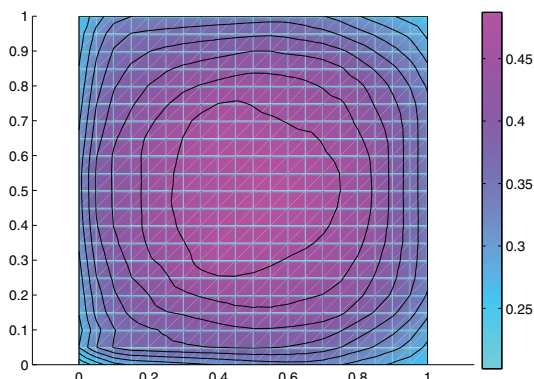

(a)

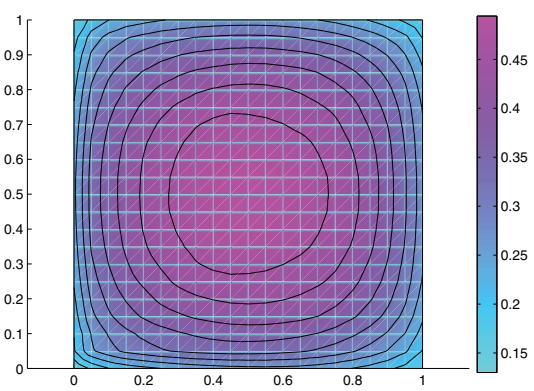

(c)

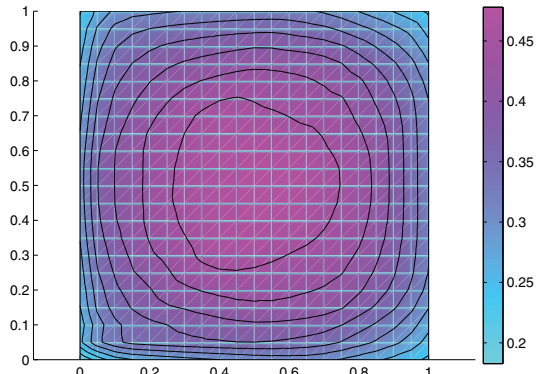

(b)

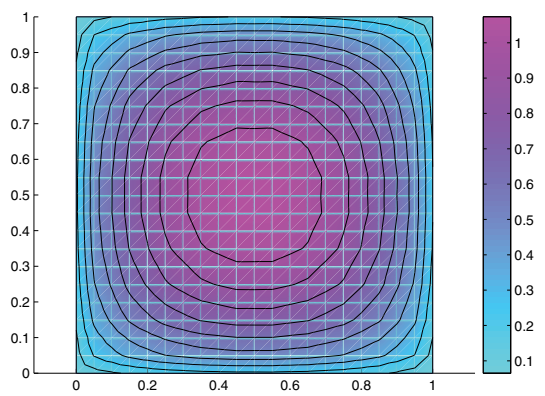

(d)

FIG. 1. Velocity contours in the cross section of a square channel: (a-d) related to $\delta=0.01,0.1,1$ and 10 .

These results are demonstrated through some plots, where in Figure 1 the velocity distributions in a rectangular channel are plotted for different values of the rarefaction parameter $\delta=0.01,0.1,1$, and 10. In Figures 2 and 4 , the $u$ level sets in a circular and an equilateral triangular channel are plotted for different values of $\gamma_{0}$ and for the rarefaction parameters $\delta=0.1$ and $\delta=1$, respectively. One may see the smoothing effects of the SD parameter on corresponding figures. In Figure 3 we also present the velocity level sets for a right-angle triangular cross section and for different values of $\delta$.

We now present a numerical example for solving the boundary value problem (2.16) and illustrate the performance of the proposed method on numerical convergence.

Example 4.1. In this example, we study problem (2.16) considering the spatial domain to be the unit square $[0,1] \times[0,1]$ and with the source function given by

$$
\begin{aligned}
S(\boldsymbol{v}, \boldsymbol{x})= & w(\theta, \boldsymbol{x})+c w(\theta, \boldsymbol{x}) \cos (\theta)(\theta y \sin (\pi x) \sin (\pi y)+\pi \theta x y \cos (\pi x) \sin (\pi y)) \\
& +c w(\theta, \boldsymbol{x}) \sin (\theta)(\theta x \sin (\pi x) \sin (\pi y)+\pi \theta x y \cos (\pi y) \sin (\pi x)) \\
& -\left(\frac{1}{2 \pi}\left(e^{2 \pi x y \sin (\pi x) \sin (\pi y)}-1\right)\right) /(x y \sin (\pi x) \sin (\pi y)),
\end{aligned}
$$

where

$$
w(\theta, \boldsymbol{x})=e^{\theta x y \sin (\pi x) \sin (\pi y)} .
$$




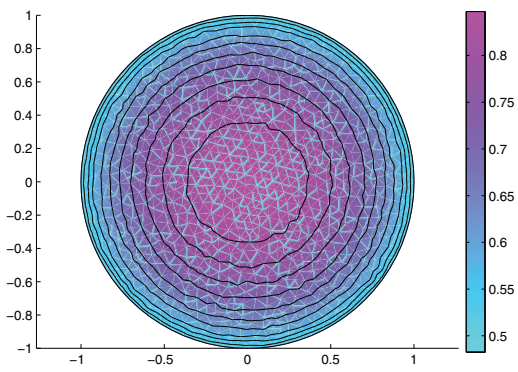

(a)

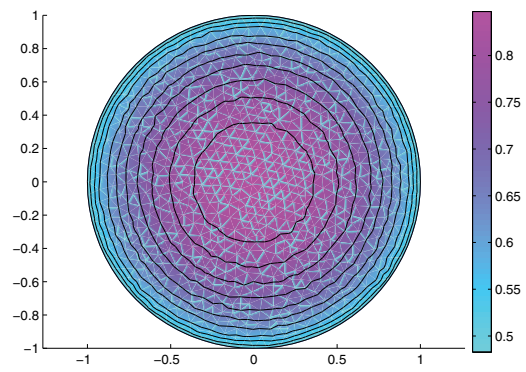

(b)

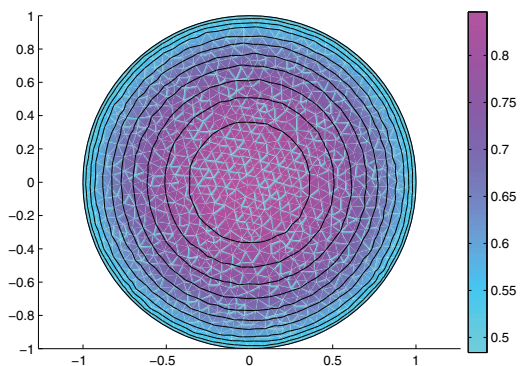

(c)

FIG. 2. Comparison of the velocity contours for the unit circle with $\delta=0.1:$ (a-c) related to $\gamma_{0}=0,0.001$ and 0.01 .

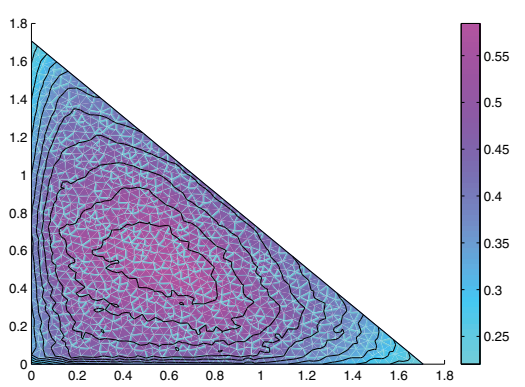

(a)

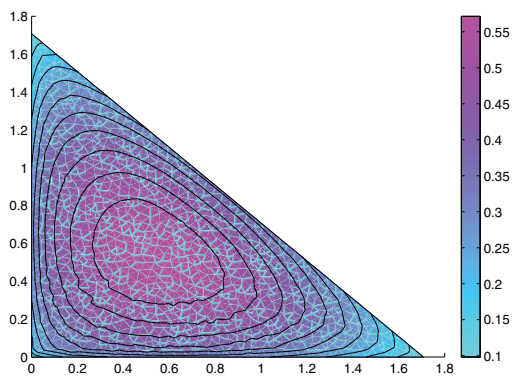

(c)

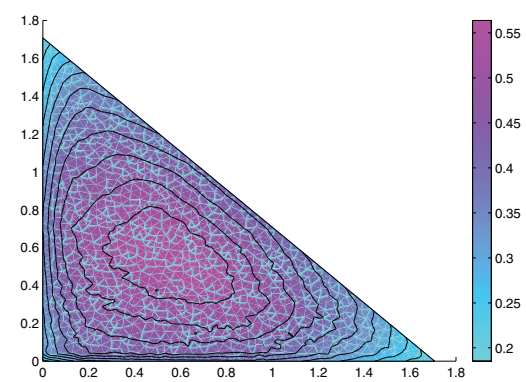

(b)

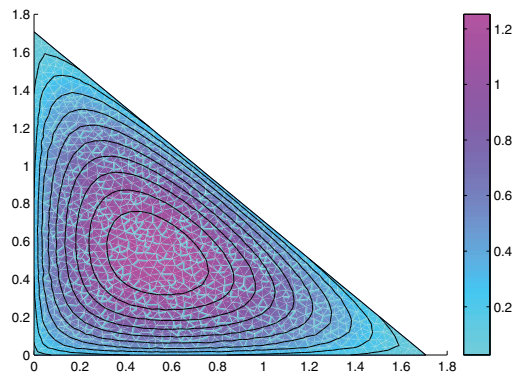

(d)

FIG. 3. Velocity contours for the orthogonal triangle: (a-d) related to $\delta=0.01,0.1,1$ and 10 . 


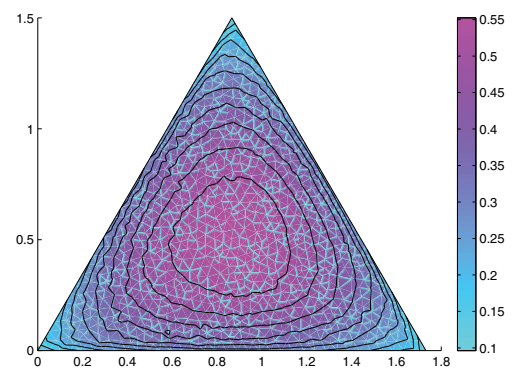

(a)

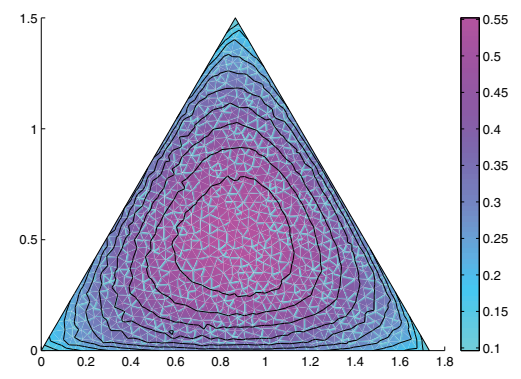

(b)

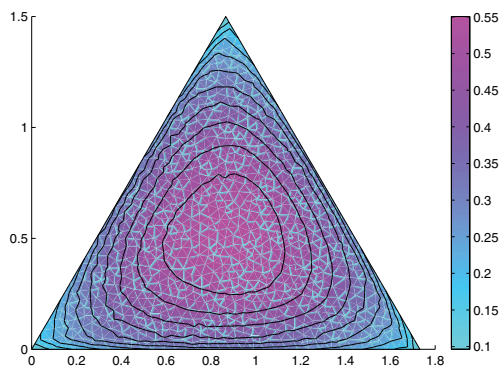

(c)

FIG. 4. Comparison of the velocity contours for the equilateral triangle with $\delta=1:(a-c)$ related to $\gamma_{0}=0,0.001$ and 0.01 .

This example is particularly designed so that the $c$ dependence present in $S$ is canceled out and the exact solution $f$ becomes independent of $c$ given by

$$
f(\boldsymbol{v}, \boldsymbol{x})=w(\theta, \boldsymbol{x})-1 .
$$

With such a choice, reducing the velocity discretization to an angular quadrature, we may focus on the study of the spatial discretization. To compute the spatial errors, we will use the standard strategy considering higher-order approximations in the quadrature rule (3.1), now only angular, and then compute the convergence order of the space discretization. In Tables 4 and 5 and Figure 5, we show these convergence results in the $L_{2}$-norm, as well as the $\||\cdot|\|$-norm defined by (3.20). We also present the error of the mass flow rate $G$, defined by (2.21), in Table 6 and Figure 6 , for the different values of $\gamma_{0}$.

To see the influence of the numerical quadrature error on the solution accuracy, we also used the less refined numerical quadrature with $M=5$ and $N=5$, i.e., only 25 ordinates. For instance, corresponding to $h=\frac{\sqrt{2}}{20}$ the errors for the numerical solution in the $L_{2}$-norm, $\left\|u-u_{h}\right\|$ for the different (see Tables 4-6) values for the parameter $\gamma_{0}$, are, respectively, 0.00681873, 0.006722791, 0.006416259, 0.009199408, and 0.033521166, and those for ||$u-u_{h}|| \mid$ are, respectively, 0.007871414, 0.007699679, $0.007173271,0.010485591$, and 0.037295534 . Thus, in this example, the errors in the numerical solution are mainly due to the spatial discretization and are of the order of optimal convergence rate, $\mathcal{O}\left(h^{3 / 2}\right)$, for piecewise linears.

We have varied $\gamma_{0}$ in the SD parameter $\gamma=\gamma_{0} h$ in (3.33) to check the influence of the stabilization parameter on the fully discrete solution. In Table 4 we see that increasing $\gamma_{0}$ further, the $L_{2}$ error increases due to smearing. 

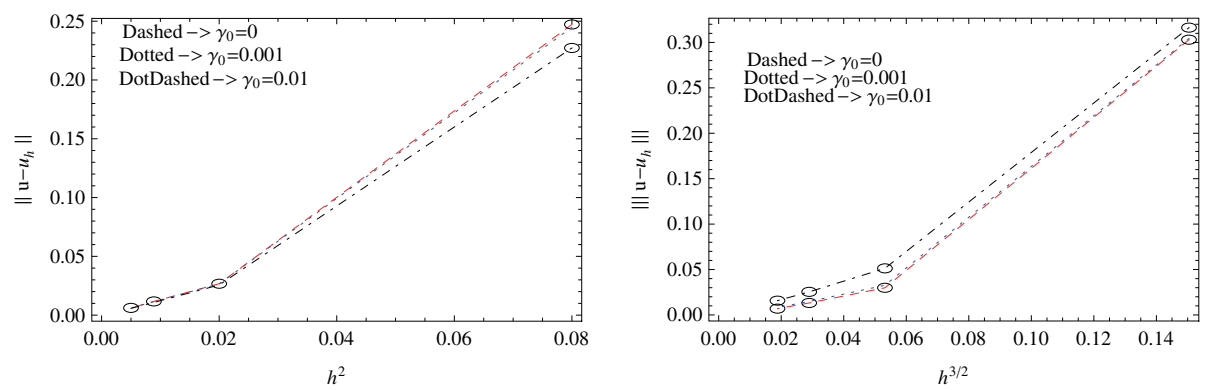

FIG. 5. $\left\|u-u_{h}\right\|$ vs. $h^{2}$ (left) and \|\|$u-u_{h} \| \mid$ vs. $h^{3 / 2}$ (right) for Example 4.1.

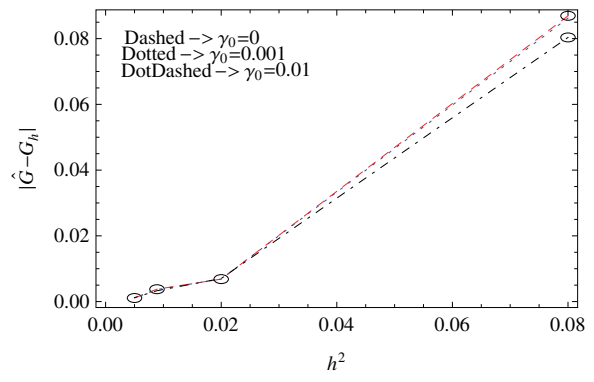

FIG. 6. $\left|G-G_{h}\right|$ vs. $h^{2}$ for Example 4.1 .

Conclusion. The fully developed flow of a rarefied gas in a channel with arbitrary cross section, due to an imposed pressure gradient, can be described by a BGK model. This paper concerns stability and convergence of a fully discrete scheme approximating the solution of a linear BGK equation. The approximation is based on a discrete-ordinates method for the velocity variable combined with the SD finite element method in the spatial domain. We derive optimal convergence rates due to the maximal available regularity of the exact solution, $\mathcal{O}\left(h^{k+1 / 2}\right)$, for piecewise continuous polynomial approximation of spectral order $k$ and with the exact solution $f$ in the Sobolev space $H^{k+1}(\Omega)$. The compatibility relation between the two discretization methods, with $m \sim n=N M$ quadrature points in velocity and the spatial finite element mesh size $h$, reads as $\mathcal{O}\left(\delta^{1 / 2} h^{k+1 / 2}\right) \sim \mathcal{O}\left(m^{-\alpha}+\delta^{1 / 2} m^{-\alpha / 2}\right)$, $\alpha \approx k+1$, and $\delta$ is the rarefaction parameter. In the numerical investigations, results for the velocity profiles and the flow rate have been provided for various geometries for the flow channel with the rectangular, circular, and triangular cross sections, in the whole range of rarefaction parameter $\delta$. The convergence rates in both the $L_{2^{-}}$ and the triple norm, $\||\cdot|\| \mid$, are justified for a generic example in a square spatial domain.

In contrast to the previously published results, the solution herein is extended easily into the transitional near continuum regime $\delta \gg 1$, e.g., for conventional finitedifference methods applied to the kinetic equation in the polar coordinate system, due to stability issues, the results been presented have only up to a rarefaction parameter $\delta=50$. In addition, it seems that the present scheme can be used to solve other physically relevant models derived from the Boltzmann equation. 


\section{REFERENCES}

[1] H. Adams, Sobolev Spaces, Academic Press, New York, 1978.

[2] M. Asadzadeh, Analysis of a fully discrete scheme for neutron transport in two-dimensional geometry, SIAM J. Numer. Anal., 23 (1986), pp. 543-561.

[3] M. Asadzadeh, Streamline diffusion methods for Fermi and Fokker-Planck equations, Transport Theory Statist. Phys., 26 (1997), pp. 319-340.

[4] P. L. Bhatnagar, E. P. Gross, and M. Krook, A model for collision processes in gases, Phys. Rev., 94 (1954), pp. 511-515.

[5] V. Bobylev and C. Cercignani, Discrete velocity models without nonphysical invariants, J. Statist. Phys., 97 (1999), pp. 677-689.

[6] A. V. Bobylev, A. Palczewski, and J. Schneider, On approximation of the Boltzmann equation by discrete velocity models, C. R. Acad. Sci. Paris Ser. I Math., 320 (1995), pp. 639-644.

[7] C. Buet, A discrete-velocity scheme for the Boltzmann operator of rarefied gas dynamics, Transport Theory Statist. Phys., 25 (1996), pp. 33-60.

[8] C. Cercignani, The Boltzmann Equation and Its Applications, Springer-Verlag, New York, 1988.

[9] C. Cercignani and A. V. Bobylev, Discrete velocity models: The case of mixtures, Transport Theory Statist. Phys., 29 (2000), pp. 209-216.

[10] P. G. Ciarlet, The Finite Element Method for Elliptic Problems, North-Holland, Amsterdam, 1978.

[11] A. Ern and J.-L. Guermond, Theory and Practice of Finite Elements, Appl. Math. Sci. 159, Springer-Verlag, New York, 2004.

[12] I. Graur AND F. Sharipov, Gas flow through an elliptical tube over the whole range of the gas rarefaction, Eur. J. Mech. B. Fluids, 27 (2008), pp. 335-345.

[13] T. J. R. Hughes And A. Brooks, A multidimensional upwind scheme with no crosswind diffusion in Finite Element Methods for Convection Dominated Flows, T. J. R. Hughes, ed., ASME, New York, 1979.

[14] T. J. R. Hughes and M. Mallet, A new finite element formulation for computational fluid dynamics III. The generalized streamline operator for multidimensional advective-diffusive systems, Comput. Methods Appl. Mech. Engrg., 58 (1986), pp. 305-328.

[15] C. Johnson And J. Saranen, Streamline diffusion methods for the incompressible Euler and Navier-Stokes equations, Math. Comp., 47 (1986), pp. 1-18.

[16] S. S. Lo AND S. K. Loyalka, An efficient computation of near-continuum rarefied gas flows, Z. Angew. Math. Phys., 33 (1982), pp. 419-424.

[17] S. K. Loyalka, T. S. Storvik, and H. S. Park, Poiseuille flow and thermal creep flow in long, rectangular channels in the molecular and transition flow regimes, J. Vac. Sci. Technol., 13 (1976), pp. 1188-1192.

[18] F. Sharipov, Rarefied gas flow through a long rectangular channel, J. Vac. Sci. Technol. A, 17 (1999), pp. 3062-3066.

[19] D. Valougeorgis And S. NARIs, Acceleration schemes of the discrete velocity method: Gaseous flows in rectangular microchannels, SIAM J. Sci. Comput., 25 (2003), pp. 534-552.

[20] D. Valougeorgis and S. NARIS, Rarefied gas flow in a triangular duct based on a boundary fitted lattice, Eur. J. Mech. B. Fluids, 27 (2008), pp. 810-822.

[21] D. Valougeorgis and J. R. Thomas, Exact numerical results for Poiseuille and thermal creep flow in a cylindrical tube, Phys. Fluids., 29 (1986), pp. 423-429. 